\title{
LOW LUMINOSITY RADIO GALAXIES
}

\author{
P. PARMA
}

Istituto di Radioastronomia del CNR via Gobetti, 101, 40129 Bologna

H.R. DE RUITER

Osservatorio Astronomico di Bologna

via Zamboni, 33, 40126 Bologna

\author{
AND \\ R. FANTI \\ Dipartimento di Fisica, Università di Bologna \\ via Irnerio, 46, 40126 Bologna
}

\section{Introduction}

This review talk will deal almost exclusively with the radio aspects of low luminosity radio galaxies, as the other wavelength bands (optical, X, IR) are covered by others during this conference. By low radio luminosity we mean a radio power at $20 \mathrm{~cm}$ in the range $10^{19}$ to $10^{24.5} \mathrm{~W} \mathrm{~Hz}^{-1}$ (Hubble constant of 100 , as throughout this talk). At the upper limit occurs the "break" in the radio luminosity (RLF) (Auriemma et al 1977) as well as the transition from Fanaroff -Riley type I to type II (Fanaroff \& Riley, 1974). This limitation to weak radio powers does not necessarily imply that such radio sources constitute a homogeneous class of objects; it has been shown by Wrobel \& Heeschen (1991) that a number of radio galaxies of very low luminosity have a ratio between far-infrared and radio emission similar to spiral galaxies and this might suggest that these objects are powered by star formation phenomena. Long baseline interferometry is able to pinpoint compact radio nuclei and therefore can distinguish starburst related radio sources from those powered by nuclear activity (see Slee et al 1994).

The aspects of low and high luminosity radio sources are very different, in fact so much so that the classification scheme proposed by Fanaroff and Riley (1974) has often been thought to reflect fundamental physical differ- 
ences. FR I and FR II are considered to be different in morphology because of different power of the nuclear engine; in addition also the environment is believed to play a role in deciding whether a source will become an FR I or FR II source.

In this talk I will review some of the old and established ideas about FR I sources, but also discuss their properties in the light of recent unification schemes.

\section{FR I sources in the eighties}

\subsection{MORPHOLOGY, JETS}

The original Fanaroff-Riley classification turned out to be quite useful; numerous high resolution observations done with the VLA from 1980 onwards have shown that there are a number of characteristic properties common to the large majority of low luminosity sources.

What is an FR I source like? Often $3 \mathrm{C} 31$, with its double jets ending in characteristic plumes, has been put forward as a kind of prototype of low luminosity sources, but in reality this is not quite true. Studies done in recent years with the VLA show that lobe-sources (and not "plumes") are the rule. The lobes are usually "fat", with an average ratio of length to width of 2:1. In the B2 sample of low luminosity radio galaxies $62 \%$ are double sources with lobes, $18 \%$ are tailed sources (WATs and NATs), 11 $\%$ compact (radio cores), and only $4 \%$ are "naked" jets (de Ruiter 1990). 3C 31 resembles more a naked jet than any other type. The naked jets occur preferably at the low end of the power range $\left(\leq 10^{23} \mathrm{~W} \mathrm{~Hz}^{-1}\right)$. Tail sources are typically a factor 10 stronger, up to the break luminosity. At the upper end of the luminosity range $\left(\sim 10^{25} \mathrm{~W} \mathrm{~Hz}^{-1}\right)$ we find sources with an overall FR I structure but with one-sided jets and hot spots in the middle of the lobes. Often the global structures are distorted. In some sources wiggles or oscillations suggest that non-uniform motion of the parent galaxy or of the power source may be responsible for this. Interaction between the outer parts of the sources and an intergalactic medium is the almost certain cause of the curved tails, which define Wide Angle Tail and Narrow Angle Tail sources, seen in galaxy clusters and groups.

Perhaps the most important characteristic is the presence, in a large majority of FR I sources, of prominent, usually rather symmetric twin jets: in the B2 sample of low luminosity radio galaxies (Parma et al 1987) as many as one half to three quarters have large scale jets that are easily detectable in VLA-type observations.

However, even though the two sides of FR I sources are very symmetric and rarely differ by more than a factor 2 in intensity, the jets tend to be 
more asymmetric the closer one looks near the nucleus (jet base). This is a very significant fact as we will see later on.

The relaxed morphology of FR I radio sources suggest low flow velocities in the large scale jets. Bicknell (1984) developed a model of turbulent low Mach number jets, where the observed subadiabatic behaviour of the jet brightness is supposed to be caused by entrainment of external material and subsequent deceleration of the jets. He convincingly showed, first for a limited number of sources and later for a larger sample of FR I sources (Bicknell et al 1990), that a low Mach number $(M \sim 2)$, turbulent jet does indeed reproduce observed jet properties. The flow velocities far from the base of the jets suggested by the application of his model are indeed low, of the order of a few hundreds of $\mathrm{km} / \mathrm{s}$ to at most $10000 \mathrm{~km} / \mathrm{s}$. Therefore large scale beaming effects should not be important.

\subsection{SIZES}

Equal power does not imply equal size, even though there exists a well known correlation between the two (de Ruiter et al 1990). The correlation, however, is very loose, and two sources with the same total luminosity may differ greatly in size. Other factors indicate that besides power also the environment must play an important role. Thus we find that if a source is distorted close to the nucleus it will not reach much beyond the size of the parent galaxy.

In order to bring the effects out more clearly we introduce a normalized linear size $l_{n}$, which is the ratio between the size of the source and the median size of sources at a given power: thus we take out the dependence of size on radio power. Using the B2 sample of low luminosity radio galaxies (de Ruiter et al 1990) we find that if a source has a large spreading rate $(>0.3)$ it remains small, $l_{n}<1$, but if the spreading rate is small, isolated galaxies tend to become big, with $l_{n}>1$. Sources in clusters or groups of galaxies have $l_{n} \leq 1$, regardless of the spreading rate. These factors listed here therefore point to the properties of the environment (both the nature of the galaxy, its gas content, and the intergalactic medium) as being another decisive factor for the size of a radio source.

\subsection{AGES}

In principle synchrotron theory provides a direct way to determine the age of the electrons responsible for the synchrotron emission.

For a number of FR I sources in the B2 and 3C samples there are now data of sufficient quality (at 6 and $20 \mathrm{~cm}$ ) to try and establish an age of the radio emitting material in the lobes. We assume that the spectral index is a direct indication of the time passed since the last acceleration, 
and use the Jaffe-Perola model (Jaffe \& Perola 1973), which assumes an isotropic redistribution of pitch angles, starting from a power-law distribution of electron energies. As usual electron energy losses are considered to be caused by the synchrotron process itself and by inverse Compton process. Taking the steepest spectral index found in the lobes (presumably the oldest region), we used the formulae of Myers \& Spangler (1985) to derive the ages $(\tau)$ of the sources, and, with the linear size, a velocity of source growth, $v_{\text {growth }} \sim D / \tau$.

Practically all ages determined this way fall in the range $10^{7}-10^{8}$ years, and no correlation with linear size is seen. This immediately implies that the velocity of growth is, statistically speaking, dependent on radio power (through the well known correlation of size and power). A similar correlation was derived by Alexander \& Leahy (1987) and Liu et al (1992), who used only $3 \mathrm{C}$ sources. The velocities are well below $10^{4} \mathrm{~km} \mathrm{~s}^{-1}$, at least for FR I sources at low redshifts.

\section{FR I sources in the nineties}

\subsection{DEPOLARIZATION}

Low luminosity sources appeared to be understood reasonably well, at least qualitatively, when some new developments arose. First, when unification schemes were realized to be quite promising, a number of authors searched for possible non-beamed counterparts of BL Lac objects. The natural candidates, as all agreed, were FR I sources (Urry \& Padovani 1995 and reference therein).

Since the large scale low luminosity jets contain slow-moving material, it is not immediately clear how this unification can come about. It was noticed, however, that the depolarization asymmetry found by Garrington et al. (1988), considered to be caused by an orientation effect plus beaming (Laing 1988), was also present in FR I sources, albeit at a much lower level of significance (de Ruiter et al. 1993). These results were in reality based on polarization data at one frequency. At present we have depolarization data and the general trend is fully confirmed: also in FR I sources there is, statistically speaking, an asymmetry in depolarization, but it should be said that this is seen only in a subset, while many other sources are quite symmetric in jet and counterjet depolarization. Therefore the effect is far less dominant than in the sources studied by Garrington et al (1988), which formed the basis of Laing's hypothesis that the depolarization asymmetry reflects beaming. Nevertheless, if the effect in FR I sources is due to the same cause, beaming should occur to some extent also in low luminosity sources.

Subsequently Laing (1993), Komissarov (1994) and Bicknell (1994) pro- 
posed a new phenomenological model, in which all jets, including those of FR I sources, are (mildly)-relativistic close to the core (at the inner kpc, or even closer in the lowest luminosity sources), and decelerate quickly to form slow-moving $\left(<10^{4} \mathrm{~km} \mathrm{~s}^{-1}\right)$ large scale jets.

\subsection{BRIGHTNESS ASYMMETRIES}

A way to substantiate this idea would be to search for brightness asymmetries in the inner parts of FR I jets. This has been done by Parma et al (1994), who found that the brightness asymmetries, as well as the distribution of core powers are consistent with mildly $(\beta \sim 0.7-0.8)$ relativistic velocities close to the core, while the jet and counterjet gradually become symmetric as the distance from the core increases.

Beyond a few kpc FR I sources are quite symmetric, as has been known for many years now, but the data suggest that' asymmetries persist longer for more powerful sources: jet deceleration may be a function of the strength of a source such that close to the transition FR I to FR II Doppler effects can be important. In fact, the residual velocities, after the initial deceleration tend to be higher in the more powerful sources. This follows from energy budget considerations (see Parma et al 1994). The difference FR I/FR II is in this scenario nothing but an effect of jet velocity, and the more powerful radio sources will take up the aspect of FR II's (jets that are asymmetric all the way, hot-spots, etc.).

The question why a source is an FR I or an FR II in the first place, is probably closely linked also with the environment: as Owen \& Ledlow (1994) have convincingly shown, the dividing line between FRI and II is a function of the absolute magnitude of the parent galaxy; it is therefore not only the intrinsic strength of the source, i.e. the quantity of energy produced by the central power source, but also the medium and the (gradient of the) gravitational potential the radio material encounters on its way out (see Bicknell 1995).

Summarizing our current ideas about low luminosity sources and unification schemes: FR I jets appear to start (mildly) relativistic, but quickly decelerate, the quicker the lower the power of the source. FR II sources on the other hand remain supersonic until they impact on the external medium and form hot spots.

Considering BL Lacs as the beamed fraction of FR I sources is then a logical consequence, although there remains some doubt as to the Lorentz factors required: in the case of BL Lacs one needs typically $\Gamma \sim 5$ (see e.g. Urry \& Padovani 1995), whereas both the jet asymmetries and the distribution of core strengths suggest $\Gamma \leq 2$. Some way will have to be found to remove this discrepancy. 


\section{Future prospects}

It is clear that our ideas about low luminosity radio sources have changed rapidly in the past few years, and this naturally suggests the direction future research will take. Especially the properties of FR I jets close to the nucleus have gained importance, and VLB type observations will have to provide new data on the detailed physics of such jets.

Another new and important development, actually taking place right at the moment, are the new "all-sky" or,-better-, "important-parts-of-thesky" surveys, carried out both with the VLA and the WSRT. They will provide catalogs of hundreds of thousands of objects to relatively low flux densities at different frequencies $(1.4 \mathrm{GHz}$ for the VLA, $327 \mathrm{MHz}$ and, partly $610, \mathrm{MHz}$ for the WSRT). Extraction of huge samples (many thousands) of galaxies from the WENSS survey are already in progress and will lead to samples of low luminosity radio galaxies that contain one to two orders of magnitude more objects than in the existing samples. Statistics will not be a problem anymore!

\section{References}

Alexander, P. \& Leahy, J.P. 1987, MNRAS, 225, 1

Auriemma, C., Perola, G.C., Ekers, R., Fanti, R., Lari, C., Jaffe, W.J., \& Ulrich, M.H. $1977, A \& A, 57,41$

Bicknell, G.V. 1984, ApJ, 286, 68

Bicknell, G.V., de Ruiter, H.R., Fanti, R., Morganti, R. Parma, P. 1990, ApJ, 354, 98

Bicknell, G.V. 1994, ApJ, 422, 542

Bicknell, G.V. 1995, ApJ, in press

de Ruiter, H.R., Parma, P., Fanti, C., Fanti, R. 1990, $A \& A$, 227, 351

de Ruiter, H.R., Capetti, S., Morganti, R., Parma, P., Lazzari, L., Fanti, R. 1993, Jets in extragalactic radio sources, eds Roser, H.-J., Meisenheimer, K., Springer-Verlag, p.21

Fanaroff, B.L. \& Riley, J.M. 1974, MNRAS, 167, 31p

Garrington, S.T., Leahy, J.P., Conway, R.G., Laing, R.A. 1988, Nature, 331, 147

Jaffe, W.J., Perola, G.C. 1973, $A \& A$, 26, 423

Komissarov, S. 1994, MNRAS, 269, 394

Laing, R.A. 1988, Nature, 331,149

Laing, R.A. 1993, Astrophysical Jets, ST Science Institute Symposium Series 6 , eds Burgarella, D., Livio, M. \& O'Dea, C.P., Cambridge University Press, p.95

Liu, R., Pooley, G., Riley, J.M. 1992, MNRAS, 269, 928

Myers, S.T. \& Spangler S.R. 1985, ApJ, 281, 52

Owen, F.N. \& Ledlow, M.J. 1994, The First Stromlo Symposium: The Physics of Active Galaxies, ASP Conference Series 54, eds Bicknell, G.V., Dopita, M. \& Quinn, P.J., p.319

Parma, P., Fanti, C, Fanti, R., Morganti, R., De Ruiter, H.R. 1987, $A \& A, 181,244$

Parma, P., de Ruiter, H.R., Fanti, R., Laing, R.A. 1994, The First Stromlo Symposium:

The Physics of Active Galaxies, ASP Conference Series 54, eds Bicknell, G.V., Dopita, M. \& Quinn, P.J., p. 21

Slee, O.B., Sadler, E.M., Reynolds, J.E., Ekers, R.D. 1994 MNRAS, 269, 928

Urry, C.M., \& Padovani, P. 1995, PASP, 107, 803

Wrobel, J.M., \& Heeschen, D.S. 1991, AJ, 101, 148 